\title{
TUSING CLINICAL PROFILES AND COMPLETE BLOOD COUNTS TO DIFFERENTIATE CAUSES OF ACUTE FEBRILE ILLNESS DURING THE 2009-2011 OUTBREAK OF TYPHOID AND CHIKUNGUNYA IN SONGKHLA, THAILAND
}

${ }^{1}$ Department of Pediatrics, ${ }^{3}$ Epidemiology Unit, Faculty of Medicine, Prince of Songkla University, Hat Yai, Songkhla, Thailand ${ }^{2}$ Department of Pediatrics, Songkhla Hospital, Songkhla, 90000, Thailand,

\section{Background and aims:}

During the recent outbreak of typhoid and chikungunya in Songkhla, Thailand an effort was made to use $\mathrm{CBCs}$ and clinical profiles to differentiate these diseases from dengue viral infection (DVI) or other acute febrile illnesses (AFIs) to enable prompt treatment.

\section{Methods:}

Previously healthy patients aged 2-15 years having fever $\leq 3$ days without localizing signs were enrolled retrospectively. Typhoid was confirmed by hemoculture, dengue by NSI or PCR, and CHIK by PCR. Febrile children negative for all 3 infections were classified as other AFI.

\section{Results:}

Of the 264 cases, 56, 24, 164, and 19 had typhoid fever, chikungunya, DVI, or other AFI, respectively. The typhoid fever group had the highest proportion of patients with diarrhea $(54 \%$ vs. $28 \%, 16 \%$, and $16 \%$ ) and duration of fever $\geq 7$ days ( $50 \%$ vs. $11 \%, 4 \%$, and $0 \%$ ) when compared to $\mathrm{DVI}$, chikungunya, and other AFI, respectively. The chikungunya group had the highest proportion of patients with arthralgia ( $96 \%$ vs. $3 \%, 0 \%$, and $10 \%)$ and rash $(84 \%$ vs. $12 \%, 0 \%$, and $13 \%)$ when compared to DVI, typhoid, and other AFI, respectively. The DVI group had the highest proportion of patients with vomiting $(70 \%)$ vs. $48 \%, 20 \%$, and $42 \%)$, hepatomegaly ( $36 \%$ vs. $4 \%, 0 \%$, and $0 \%$ ), platelets $\leq 100,000$ cells $/ \mathrm{mm}^{3}(23 \%$ vs. $9 \%, 0 \%$, and $10 \%)$, and WBCs $\leq 5,000$ cells $/ \mathrm{mm}^{3}(73 \%$ vs. $14 \%, 28 \%$, and $16 \%$ ) when compared to typhoid, chikungunya and other AFI, respectively (Tables 1 , 2).

Children who presented within the first 3 day of fever who had headache, nausea/vomiting,

hepatomegaly, hematocrit $\geq 39 \%$, WBCs $<5,000$ cells $/ \mathrm{mm}^{3}$, and platelets $\leq 100,000$ cells $/ \mathrm{mm}^{3}$ were likely to have DVI.

Children with arthralgia, rash and normal $\mathrm{CBCs}$ were likely to have chikungunya. Typhoid fever patients commonly had $\mathrm{BT}>39.5^{\circ} \mathrm{C}$, diarrhea and normal $\mathrm{CBC}$ s wen compared to children with the other diseases

\section{Conclusion:}

Careful assessment of certain clinical and CBC parameters can help the physician differentiate between these diseases with quite high reliability.

\begin{tabular}{|c|c|c|c|c|c|}
\hline Characteristic & $\begin{array}{l}\text { Typhoid } \\
(N=56)\end{array}$ & $\begin{array}{c}\text { DVI } \\
(N=165)\end{array}$ & $\begin{array}{l}\text { Chikungunya } \\
\qquad(\mathrm{N}=25)\end{array}$ & $\begin{array}{l}\text { Other AFIs } \\
\qquad(N=19)\end{array}$ & $p$ \\
\hline Male, n (\%) & $24(42.9)$ & $91(55.2)$ & $13(52.0)$ & $14(73.7)$ & 0.12 \\
\hline Age, years, mean \pm SD & $7.3 \pm 3.2^{\mathrm{a}}$ & $9.9 \pm 3.1^{b}$ & $10.2 \pm 2.3^{b}$ & $7.5 \pm 3.9^{a}$ & $<0.01$ \\
\hline Headache, n (\%) & $14 / 45(31.1)^{a}$ & $114 / 155(73.5)^{b}$ & $18(72.0)^{\mathrm{b}}$ & $6(31.6)^{\mathrm{a}}$ & $<0.01$ \\
\hline Myalgia, $n(\%)$ & $2 / 45(4.4)^{\mathrm{a}}$ & $57 / 155(36.8)^{b}$ & $21(84.0)^{\mathrm{c}}$ & $7(36.8)^{b}$ & $<0.01$ \\
\hline Arthralgia, $\mathbf{n}(\%)$ & $0^{\mathrm{a}}$ & $4 / 155(2.6)^{a}$ & $24(96.0)^{\mathrm{b}}$ & $3(15.3)^{\mathrm{c}}$ & $<0.01$ \\
\hline Abdominal pain, $\mathbf{n}(\%)$ & $17 / 45(37.8)^{\mathrm{a}}$ & $61 / 155(39.4)^{\mathrm{a}}$ & $7(28.0)^{a, b}$ & $1(5.3)^{\mathrm{b}}$ & 0.02 \\
\hline Diarrhea, $\mathbf{n}(\%)$ & $30(53.6)^{a}$ & $47(28.5)^{b}$ & $4(16.0)^{b}$ & $3(15.8)^{\mathrm{b}}$ & $<0.01$ \\
\hline Nausea/vomiting, $\mathbf{n}(\%)$ & $27(48.2)^{a}$ & $115(69.7)^{\mathrm{b}}$ & $5(20.0)^{c}$ & $8(42.1)^{\mathrm{a}}$ & $<0.01$ \\
\hline URI, n (\%) & $24(42.9)^{a}$ & $32(19.4)^{b}$ & $4(16.0)^{b}$ & $11(57.9)^{a}$ & $<0.01$ \\
\hline
\end{tabular}

$A, b, c$ Values within rows not having a superscript in common differ significantly $(p<0.05)$.

Table 2 Complete blood counts in children with fever $\leq 3$ days with typhoid fever, dengue viral infection (DVI), chikungunya or other acute febrile illness (AFIs)

\begin{tabular}{|c|c|c|c|c|c|}
\hline CBC & $\begin{array}{l}\text { Typhoid } \\
(\mathrm{N}=56)\end{array}$ & $\begin{array}{c}D V I \\
(N=165)\end{array}$ & $\begin{array}{l}\text { Chikungunya } \\
\qquad(\mathrm{N}=25)\end{array}$ & $\begin{array}{l}\text { Other AFIs } \\
(N=19)\end{array}$ & $\mathrm{p}$ \\
\hline $\begin{array}{l}\text { Hematocrit levels, } \% \text {, } \\
\text { mean +/-SD }\end{array}$ & $36.5 \pm 4.0^{\mathrm{a}}$ & $38.9 \pm 4.0^{b}$ & $37.9 \pm 3.6^{a}$ & $37.1 \pm 2.5^{\mathrm{a}}$ & $<0.01$ \\
\hline Hematocrit $\geq 39 \%, n(\%)$ & $19(33.9)^{a}$ & $88(53.3)^{b}$ & $7(28.0)^{a}$ & $6(31.6)^{a, b}$ & $<0.01$ \\
\hline $\begin{array}{l}\text { WBC } \times 10^{3} \mathrm{cells} / \mathrm{mm}^{3} \text {, } \\
\text { median (IQR) }\end{array}$ & $6.7(5.4,8.9)^{a}$ & $3.6(2.6,5.2)^{b}$ & $6.1(4.3,9.3)^{\mathrm{a}}$ & $11.7(7.1,16.7)^{c}$ & $<0.01$ \\
\hline WBC $\leq 5,000$ cells $/ \mathrm{mm}^{3}, \mathrm{n},(\%)$ & $8(14.3)^{a}$ & $120(72.7)^{a}$ & $7(28.0)^{a}$ & $3(15.8)^{a}$ & $<0.01$ \\
\hline $\begin{array}{l}\text { Neutrophils, } \% \text {, } \\
\text { mean } \pm \text { SD }\end{array}$ & $63.4 \pm 13.2^{\mathrm{a}}$ & $62.6 \pm 14.8^{a}$ & $68.6 \pm 13.8^{a, b}$ & $73.8 \pm 12.4^{b}$ & 0.04 \\
\hline $\begin{array}{l}\text { Platelets } \times 10^{3} \mathrm{cells} / \mathrm{mm}^{3} \text {, median } \\
\text { (IQR) }\end{array}$ & $185(141,230)^{a}$ & $142(101,185)^{b}$ & $251(210,302)^{\mathrm{c}}$ & $233(201,280)^{\mathrm{c}}$ & $<0.01$ \\
\hline Platelets $\leq 10^{5}$ cells $/ \mathrm{mm}^{3}, \mathrm{n},(\%)$ & $5(8.9)^{\mathrm{a}}$ & $38(23.0)^{b}$ & $0^{a}$ & $1(5.3)^{a, b}$ & $<0.01$ \\
\hline
\end{tabular}

